\title{
Clinicopathologic and molecular genetic characterization of low-grade fibromyxoid sarcoma, and cloning of a novel FUS/CREB3L1 fusion gene
}

\author{
Fredrik Mertens ${ }^{1}$, Christopher DM Fletcher ${ }^{2}$, Cristina R Antonescu ${ }^{3}$, Jean-Michel Coindre ${ }^{4}$, \\ Maurizio Colecchia ${ }^{5}$, Henryk A Domanski ${ }^{6}$, Erinn Downs-Kelly ${ }^{7}$, Cyril Fisher ${ }^{8}$, \\ John R Goldblum ${ }^{7}$, Louis Guillou ${ }^{9}$, Robin Reid ${ }^{10}$, Juan Rosai ${ }^{5}$, Raf Sciot ${ }^{11}$, \\ Nils Mandahl ${ }^{1}$ and Ioannis Panagopoulos ${ }^{1}$ \\ ${ }^{1}$ Department of Clinical Genetics, Lund University Hospital, Lund, Sweden; ${ }^{2}$ Department of Pathology, \\ Brigham and Women's Hospital, Boston, MA, USA; ${ }^{3}$ Department of Pathology, Memorial Sloan-Kettering \\ Cancer Center, New York, NY, USA; ${ }^{4}$ Department of Pathology, Institut Bergonié and University Victor \\ Segalen, Bordeaux, France; ${ }^{5}$ Department of Pathology, Istituto Nazionale Tumori, Milan, Italy; ${ }^{6}$ Department \\ of Pathology and Cytology, Lund University Hospital, Lund, Sweden; ${ }^{7}$ Department of Anatomic Pathology/ \\ L25, The Cleveland Clinic Foundation, Cleveland, OH, USA; ${ }^{8}$ Department of Pathology, Royal Marsden \\ Hospital, London, UK; ${ }^{9}$ Department of Pathology, Lausanne University Hospital, Lausanne, Switzerland; \\ ${ }^{10}$ Department of Pathology, Western Infirmary, Glasgow, UK and ${ }^{11}$ Department of Pathology, University of \\ Leuven, Leuven, Belgium
}

\begin{abstract}
Low-grade fibromyxoid sarcoma (LGFMS) is an indolent, late-metastasizing malignant soft-tissue tumor that is often mistaken for either more benign or more malignant tumor types. Cytogenetic analyses have identified a recurrent balanced translocation $\mathrm{t}(7 ; 16)(\mathrm{q} 32-34 ; \mathrm{p} 11)$, later shown by molecular genetic approaches to result in a FUS/CREB3L2 fusion gene. Whereas preliminary studies suggest that this gene rearrangement is specific for LGFMS, its incidence in this tumor type and the possible existence of variant fusion genes have not yet been addressed. For this purpose, a series of potential LGFMS were obtained from nine different soft-tissue tumor centres and subjected to molecular analysis as well as careful histopathologic review. Reverse transcriptasepolymerase chain reaction analysis disclosed a FUS/CREB3L2 fusion transcript in 22 of the $23(96 \%)$ cases that remained classified as LGFMS after the histologic re-evaluation and from which RNA of sufficient quality could be extracted, whereas none of the cases that were classified as other tumor types was fusion-positive. In one of the tumors with typical LGFMS appearance, we found that FUS was fused to the CREB3L1 gene instead of CREB3L2. The proteins encoded by these genes both belong to the same basic leucine-zipper family of transcription factors, and display extensive sequence homology in their DNA-binding domains. Thus, it is expected that the novel FUS/CREB3L1 chimera will have a similar impact at the cellular level as the much more common FUS/CREB3L2 fusion protein. Taken together, the results indicate that virtually all LGFMS are characterized by a chimeric FUS/CREB3L2 gene, and that rare cases may display a variant FUS/CREB3L1 fusion. Laboratory Investigation (2005) 85, 408-415, advance online publication, 10 January 2005; doi:10.1038/labinvest.3700230
\end{abstract}

Keywords: FUS/CREB3L1; FUS/CREB3L2; low-grade fibromyxoid sarcoma; RT-PCR

Low-grade fibromyxoid sarcoma (LGFMS) is a malignant soft-tissue tumor, first described as a

Correspondence: Professor F Mertens, MD, PhD, Department of Clinical Genetics, Lund University Hospital, SE-221 85 Lund, Sweden.

E-mail: fredrik.mertens@klingen.lu.se

Received 26 October 2004; revised and accepted 23 November 2004; published online 10 January 2005 separate entity by Evans $^{1}$ in 1987. Despite a relatively bland histologic appearance, metastases develop in about $10 \%$ of the cases, and LGFMS is often confused with more benign as well as more malignant tumor types., ${ }^{2,3}$ The incidence is presumed to be low, but it seems quite probable that these lesions have been under-recognized and LGFMS may be more common than previously appreciated. ${ }^{4}$ The spectrum of genetic rearrangements 
in LGFMS is still poorly investigated, but chromosome banding analyses have revealed a, so far, pathognomonic balanced translocation $t(7 ; 16)$ (q32-34;p11) as a recurrent feature. ${ }^{4-7}$ Further molecular cytogenetic and molecular genetic analyses of this translocation led to the identification of a novel FUS/CREB3L2 fusion gene, consisting of the $5^{\prime}$ part of the FUS gene in chromosome arm 16p and the $3^{\prime}$ part of the CREB3L2 gene (also known as BBF2H7) in $7 \mathrm{q}^{7}$ A further study of 59 low-grade soft-tissue tumors provided results indicating that this fusion gene is specific for LGFMS; all 12 fusionpositive cases in that series fulfilled the morphologic criteria for LGFMS, suggesting that reverse transcriptase-polymerase chain reaction (RT-PCR) analysis may be a valuable tool in the differential diagnosis. ${ }^{4}$ However, only three of the 12 fusionpositive cases in that series had been diagnosed as LGFMS prior to the identification of the fusion gene; the routine histopathologic diagnosis in the other nine cases ranged from fibroma to malignant fibrous histiocytoma. Thus, the possible existence of genetic heterogeneity in LGFMS, that is, to what extent LGFMS may be fusion gene-negative or associated with alternative fusion genes, has not yet been examined.

The aims of the present study were thus to evaluate the incidence of the FUS/CREB3L2 fusion gene in a series of reproducibly diagnosed cases of LGFMS, and to search for alternative pathogenetic events in FUS/CREB3L2-negative cases.

\section{Materials and methods}

\section{Tumor Samples}

Samples from 37 soft-tissue tumors diagnosed as LGFMS or potential LGFMS were retrieved from nine different tumor centers in Europe and the USA for RNA extraction and RT-PCR analysis. Frozen samples, stored at $-80^{\circ} \mathrm{C}$, were preferred, as such material would enable further genetic analyses. However, frozen samples could be obtained in only 29 cases. From the remaining eight cases, the analysis had to be restricted to RNA extracted from paraffin-embedded tissue. From all 37 cases, representative slides and blocks were sent to one of us (CDM Fletcher) in order to obtain a standardized and consistent histopathologic review of all tumors. The review was performed blinded with respect to the results of the RT-PCR analyses, and vice versa. Diagnostic criteria were as previously described..$^{2,3,8,9}$ Specifically, each tumor was characterized by alternating fibrous and myxoid areas, often with abrupt transition, with a predominantly swirling growth pattern and uniform nuclear morphology. Nuclear pleomorphism and hyperchromasia were not seen and any fascicular areas were limited in extent. The clinical, histopathologic, and cytogenetic features of cases 1818/04 and 1820/04, as well as the clinical, histopathologic, cytogenetic, and molecular genetic findings in cases 1940/03, 1941/ 03, and 1942/03, have been reported before (cases 2 and 1 in Reid et $a l^{6}$ and cases 9,8 , and 12 in Panagopoulos et al, ${ }^{4}$ respectively). Cytogenetic information from short-term cultured tumor cells was also available from two previously unreported cases (3127/03 and 1838/04), both showing the $\mathrm{t}(7 ; 16)(\mathrm{q} 32-34 ; \mathrm{p} 11)$ as the sole anomaly.

\section{Immunohistochemical Analysis}

Immunohistochemical studies were performed in all cases using the Envision Plus detection system (Dako, Carpinteria, CA, USA) according to the manufacturer's specifications. The antibodies used were S-100 protein (1:3000, polyclonal, Dako), smooth muscle actin (SMA) (1:20,000, clone IA4, Sigma, St Louis, MO, USA), glial fibrillary acidic protein (GFAP) (1:8500, polyclonal, Dako), CD34 (1:400; clone Qbend10, Dako), epithelial membrane antigen (EMA) (1:200; clone E29, Dako) and desmin (1:500; clone D33, Dako). Appropriate positive and negative controls were used throughout.

\section{RT-PCR and Sequence Analysis}

RNA was extracted from frozen tissue according to standard procedures. For RNA extraction from paraffin-embedded tissue, $0.5 \mathrm{ml}$ xylene was added to the paraffin curls, vortexed for $5 \mathrm{~min}$ until the paraffin was dissolved, and spun down in a microcentrifuge for $5 \mathrm{~min}$. The xylene was removed and the first step was repeated. The tissue was then washed twice in $0.6 \mathrm{ml} 100 \%$ cold ethanol and spun down for $5 \mathrm{~min}$ at high speed, followed by vacuumdrying for $3 \mathrm{~min}$, and overnight digestion at $55^{\circ} \mathrm{C}$ in $0.48 \mathrm{ml}$ RNA digestion buffer $(20 \mathrm{mM}$ Tris $\mathrm{pH} 7.5$, $20 \mathrm{mM}$ EDTA, $1 \%$ SDS in DEPC water) and $20 \mu \mathrm{l}$ of $25 \mu \mathrm{g} / \mu$ l Proteinase K. The volume was split into two tubes and $1 \mathrm{ml}$ Trizol (Gibco BRL Inc., Gaithersburg, MD, USA) was added to each tube. Then, $200 \mu \mathrm{l}$ of chloroform was added to each tube, mixed well by vortexing and then spun down at high speed for $10 \mathrm{~min}$ at room temperature. The top aqueous RNA layer was removed and an equal volume of isopropanol was added. After at least $2 \mathrm{~h}$ at $-20^{\circ} \mathrm{C}$, the content was spun down for $10 \mathrm{~min}$, and the supernatant was removed followed by washing in $70 \%$ ethanol. The dry pellet was left for 15-20 min at room temperature and then $30-50 \mu \mathrm{l}$ of DEPC $\mathrm{H}_{2} \mathrm{O}$ was added depending of the size of the pellet. The RNA is measured using an OD ratio of $1.6-1.8$ as acceptable. The adequacy of the extracted RNA was assessed by RT-PCR, using primers for PGK transcripts. Only cases with a visible PCR product on the agarose gel were selected for further analysis.

A detailed description of the RT-PCR protocols for analysis of FUS/CREB3L2 transcripts has been reported before. ${ }^{4,7}$ In brief, a one-step PCR was performed for the amplification of the FUS/ 
CREB3L2 chimeric transcript by use of the TLS165F and BBF2-1435R primer combination. In case 1453/04, this analysis was followed by nested PCR with primers TLS263F (sequence ATATGGCTC GACTGGCGGCTATGG) and BBF2-1396R. To confirm the presence of a FUS/CREB3L1 fusion gene, RT-PCR with primer combination TLS165F and OASIS-898R (sequence TCAGCAATCAGGGTCCGC TTCTCC) was performed. Amplified fragments were sequenced according to the dideoxy procedure with an ABI Big-Dye terminator 1.1 cycle sequencing kit (Applied Biosystems, Foster City, CA, USA) with various primers on an Applied Biosystems Model 3100-Avant DNA sequencing system. BLAST software (http://www.ncbi.nlm.nih.gov/BLAST/) was used for computer analysis of sequence data.

\section{Results}

RNA of sufficient quality for RT-PCR analysis could be extracted from $26 / 29$ frozen samples and from $3 / 8$ of the paraffin-embedded tissue samples. Below follows a description of the histopathologic, clinical, and molecular genetic features of the 29 cases that were available for genotype-phenotype correlations.

\section{Histopathologic Review}

At histopathologic review, 23 of the 29 cases that could be used for RT-PCR analysis were considered to have diagnostic features of LGFMS (Table 1, Figure 1). Among these cases, one contained giant collagen rosettes and three (one of which was a local recurrence) showed foci of increased cellularity. There was no correlation between the type of FUS/ CREB3L2 transcript and tumor morphology. Similarly, the case with a variant FUS/CREB3L1 fusion gene was histologically indistinguishable from the other cases. Immunohistochemical staining in these tumors revealed the following positive results: S-100 protein 1/23 cases (weak, focal), SMA 1/23 cases (focal), CD34 2/21 cases, desmin 1/6 cases (focal), GFAP 0/21 cases and EMA 10/23 cases (3 being very focal). The frequency of EMA positivity mirrored personal unpublished observations (CDMF) in larger case numbers, but this antigen has not been examined previously in a large series. The significance of this result is uncertain.

Of the six tumors that did not fulfill the morphologic criteria for LGFMS, four were classified as myxoid spindle cell neoplasm/sarcoma not otherwise specified, one as cellular myxoma, and one as possible LGFMS (data not shown). Principal reasons for exclusion were either the presence of a

Table 1 Clinicopathologic and molecular genetic data on 22 FUS/CREB3L2-positive and one FUS/CREB3L1-positive LGFMS ${ }^{\text {a }}$

\begin{tabular}{|c|c|c|c|c|c|}
\hline Case no. & Age/sex & Site & Size & Follow-up $p^{\mathrm{b}}$ & $R T-P C R^{\mathrm{c}}$ \\
\hline 1940/03 & $\mathrm{F} / 37$ & Thigh & 12 & R 155, NED 156 & ex7/ex5 \\
\hline 1941/03 & $\mathrm{M} / 34$ & Lower back & 9 & R 24, M 48 and 60, LTF 60 & ex6/ex5 \\
\hline 1942/03 & $\mathrm{M} / 53$ & Retroperitoneal & 18 & R 70, LTF 70 & ex7/ex5 \\
\hline $3127 / 03$ & $\mathrm{M} / 39$ & Thigh & 5 & NED 9 & ex5/ex6 \\
\hline $3845 / 03$ & $\mathrm{M} / 75$ & Axilla & 7 & NED 96 & ex6/ex5 \\
\hline $1076 / 04$ & $\mathrm{~F} / 21$ & Trunk wall & ND & M 12 years, R 14 and 20 years, LTF 20 years & ex6/ex5 \\
\hline $1160 / 04$ & $\mathrm{M} / 40$ & Hip & 9 & NED 48 & ex5/in4 \\
\hline $1161 / 04$ & $\mathrm{M} / 27$ & Parascapular & ND & R+M 23 years, NED 24 years & in6/ex5 \\
\hline $1162 / 04$ & $\mathrm{~F} / 36$ & Leg & 6 & NED 67 & ex5/in5 \\
\hline $1351 / 04$ & $\mathrm{~F} / 28$ & Thigh & 11 & NED 28 & ex6/ex5 \\
\hline $1453 / 04$ & $\mathrm{M} / 42$ & Thoracic wall & 7 & NED 94 & ex9/ex5 \\
\hline $1454 / 04$ & $\mathrm{~F} / 13$ & Thigh & ND & $\mathrm{R} 13,16,19,21,22$, and 23 years NED 27 years & ex6/ins/ex5 \\
\hline $1455 / 04$ & $\mathrm{~F} / 33$ & Buttock & 6 & NED 21 & ex6/ex5 \\
\hline $1818 / 04$ & $\mathrm{~F} / 18$ & Thigh & 5 & NED 44 & ex5/in5, ex6/in5 \\
\hline $1820 / 04$ & $\mathrm{M} / 44$ & Buttock & 4 & NED 24 & in6/ex5 \\
\hline $1838 / 04$ & $\mathrm{M} / 38$ & Thigh & 9 & NED 2 & ex7/ex5 \\
\hline $1905 / 04$ & $\mathrm{~F} / 12$ & Buttock & 3 & LTF & ex6/ex5 \\
\hline PB2 & $\mathrm{M} / 12$ & Foot & 3 & R 19, NED 36 & ex6/ex5 \\
\hline PB4 & $\mathrm{F} / 41$ & Brachial plexus & ND & R 40, NED 48 & ex7/ex5 \\
\hline PB5 & $\mathrm{F} / 25$ & Neck & 6 & R+M 156, NED 252 & ex7/ex5 \\
\hline PB8 & $\mathrm{M} / 30$ & Foot & ND & Rx2, M 60, AwD 204 & ex6/ex5 \\
\hline PB11 & $\mathrm{M} / 37$ & Thigh & 9 & M 21, 32, AwD 120 & ex6/ex5 \\
\hline PB13 & $\mathrm{F} / 28$ & Thigh & 14 & NED 1 & ex7/ex5 \\
\hline
\end{tabular}

${ }^{\mathrm{a}}$ The clinicopathologic and molecular genetic features of cases 1940/03, 1941/03 and 1942/03 have been reported before (Cases 9 , 8, and 12, respectively, in Panagopoulos et $a l^{4}$ ). The clinicopathologic features of cases 1818/04, and 1820/04 have been reported before (Cases 2 and 1 in Reid et $\left.a l^{6}\right)$. RNA for RT-PCR was derived from the primary lesion in 14 cases, from a local recurrence in six cases (1940/03, 1941/03, 1076/04, 1454/04, PB4 and PB5), and from a metastasis in three cases (1161/04, PB8, PB11).

${ }^{b}$ Follow-up time in months, unless otherwise specified. $\mathrm{R}=$ local recurrence; $\mathrm{NED}=$ no evidence of disease; $\mathrm{M}=$ metastasis; $\mathrm{LTF}=$ lost to followup; AwD = alive with disease.

${ }^{\mathrm{C}}$ Location of the breakpoints in the FUS and CREB3L2 genes, or, in case 1453/04, FUS and CREB3L1, as determined by direct sequencing of fusion transcripts detected by RT-PCR. For detailed information on the localization of the breakpoints, see Figure 2. Ex=exon; in=intron; ins $=$ insertion 

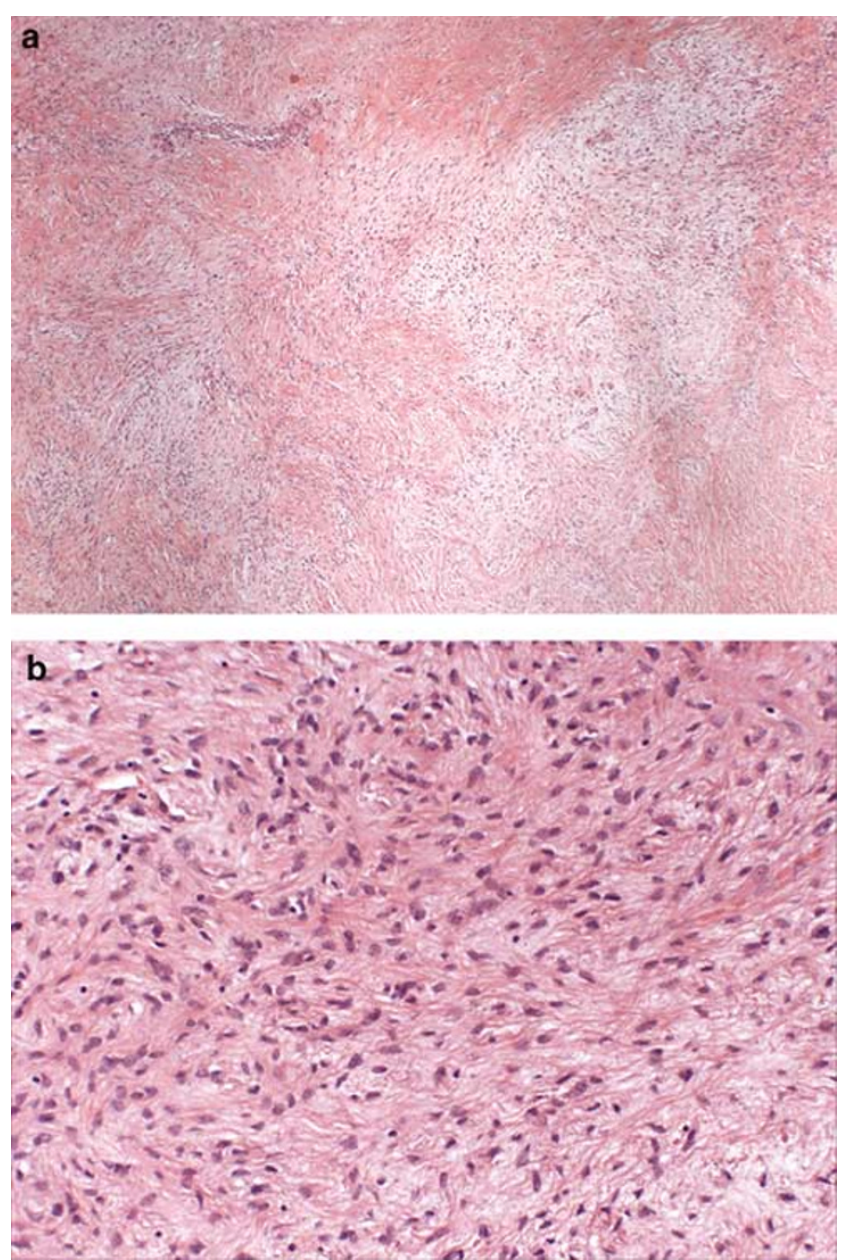

Figure 1 (a) At low power, LGFMS shows alternating fibrous and myxoid areas with a swirling growth pattern. (b) At higher power, the tumor cells have uniform spindle cell morphology with minimal nuclear atypia.

diffusely fascicular architecture or a diffusely myxoid or collagenous stroma without alternating areas.

\section{Clinical Features of the LGFMS}

The 23 cases of LGFMS (12 men, 11 women, mean age 33 years, age range 12-75 years) were all deepseated, with 15 originating in the lower extremities, three in the neck or proximal upper extremities, four in the trunk wall, and one in the retroperitoneum (Table 1). The size (largest diameter) of the primary tumor was unknown in five cases, but varied in the others from 3 to $18 \mathrm{~cm}$ (median, $7 \mathrm{~cm}$ ). Information on clinical outcome was available for 22 of the cases, with follow-up periods ranging from 1 month to 24 years.

At the time of this study, 11 patients were alive and disease-free and had not had any relapse 1-96 months after resection of the primary tumor. In four patients, the tumor had recurred locally once, after an interval of 19-155 months. In the remaining seven patients, the disease course was more complex. One patient (case 1454/04) was first operated on at the age of 13 years for what was then thought to be fibromatosis. After 13 years, there was a local recurrence, followed by another five recurrences, all of which were locally resected, after $3,6,8,9$, and 10 years. The patient has now been disease-free for 4 years.

The tumor metastasized in six patients. One (case 1941/03) developed metastases in the forearm and lungs 4 and 5 years, respectively, after diagnosis, and was then lost to follow-up. One patient (case 1161/04) was diagnosed with a parascapular tumor at the age of 27 years. After 23 years, he presented with a $4.5 \mathrm{~cm}$ local recurrence as well as a $4 \mathrm{~cm}$ lung metastasis, both of which were resected. The patient has now been disease-free for 10 months. One patient (case 1076/04) presented with a primary tumor of the abdominal wall at the age of 21 years. After 12 years, she developed multiple bilateral pulmonary metastases, followed by local recurrences after another 2 and 8 years, respectively. All tumors were surgically removed, and the patient was lost to follow-up after excision of the last relapse. One patient (case PB8) had two local recurrences before presenting with lung metastases, 60 months after the diagnosis of a primary tumor in the foot. After 12 years, he is alive with remaining disease. One patient (PB5) with a primary tumor in the posterior neck had a local recurrence and lung metastasis 156 months after diagnosis, but has since then been disease-free for 8 years. Finally, one patient developed soft-tissue and lung metastases 21 and 32 months after diagnosis, and is currently alive with remaining tumor burden.

\section{RT-PCR Findings}

RT-PCR analysis with primers specific for the FUS and CREB3L2 genes revealed a FUS/CREB3L2 fusion transcript in 22/29 cases, all of which fulfilled the morphologic criteria for LGFMS (Table 1, Figure 2). Direct sequencing of the chimeric transcripts revealed that the breakpoint in CREB3L2 was located in exon 5 in 18 cases, in intron 5 in two cases, and in intron 4 and exon 6, respectively, in one case each. The breakpoint in the FUS gene was more variable, three being located in exon 5, 10 in exon 6, two in intron 6, and six in exon 7. In addition, one case (1818/04) displayed two fusion transcripts; one showing a junction between exon 5 of FUS with intron 5 of $C R E B 3 L 2$, and another aligning FUS exon 6 with CREB3L2 intron 5. Finally, in one of the cases (1454/04), there was a small insertion at the junction. The by far most common types of chimeric transcript aligned exon 6 or 7 of FUS with exon 5 of $C R E B 3 L 2$, seen in 10 and six cases, respectively.

In one case (1453/04), RT-PCR analysis with the primer combination TLS165F and BBF2-1435R 
FUS exon 6 (entire) $\quad \boldsymbol{\nabla}$ CREB3L2 exon 5

1076-04 CCCAGAGGTCGTGGAGGTGGCCGTGGAGGCAGAGGTGGCATGGGAGACTCAGAGGGCAGCCTGAGTCCCAACCCACGCCTGCACCCCTTC $\begin{array}{llllllllllllllllllllllllllllllllll}P & R & G & R & G & G & G & R & G & G & R & G & G & M & G & D & S & E & G & S & L & S & P & N & P & R & L & H & P & F\end{array}$

FUS exon $5 \boldsymbol{\nabla} \quad$ CREB3L2 intron 4 (inverted) $\quad$ CREB3L2 exon 5

1160-04 AGCAGTGGTGGTGGAGGTGGAGGTGTGGTCCACTGGGGCTGCAAAAAAGGAAGCACGAGTCCCAACCCACGCCTGCACCCCTTCAGCCTG $\begin{array}{llllllllllllllllllllllllllllllll}S & S & G & G & G & G & G & G & V & V & H & W & G & C & K & K & G & S & T & S & P & N & P & R & L & H & P & F & S & L\end{array}$

FUS exon $6 \quad \boldsymbol{\nabla}$ FUS intron $6 \quad \boldsymbol{\nabla}$ CREB3L2 exon 5

1161-04 GGTGGCCGTGGAGGCAGAGGTGGCATGGGCTTCCAGGAATTGGCTACTCCACGCCTGCACCCCTTCAGCCTGCCTCAGACCCACAGCCCC $\begin{array}{llllllllllllllllllllllllllllllllll}G & G & R & G & G & R & G & G & M & G & F & Q & E & L & A & T & P & R & L & H & P & F & S & \text { L } & P & Q & T & H & S & P\end{array}$

FUS exon 5 CREB3L2 intron 5 CGTGGAGGCCGCGCAGTGGCTTCTTCAGTGACTTTTCAAAATACTGCCTTTTGCTTAGAATGCACTCCTCTCTCTTATAAACTGCAGGGATCA $\begin{array}{llllllllllllllllllllllllllllllllll}R & G & G & R & A & V & A & S & S & V & T & F & Q & N & T & A & F & C & L & E & C & T & P & L & S & Y & K & L & Q & G & S\end{array}$

\section{FUS exon 6 (entire) $\quad \boldsymbol{V} \quad$ CREB3L2 exon 5}

1351-04 CCAGAGGTCGTGGAGGTGGCCGTGGAGGCAGAGGTGGCATGGGTGACTCAGAGGGCAGCCTGAGTCCCAACCCACGCCTGCACCCCTTCA $\begin{array}{lllllllllllllllllllllllllllllll}R & G & R & G & G & G & R & G & G & R & G & G & M & G & D & S & E & G & S & L & S & P & N & P & R & \text { L } & H & P & F\end{array}$

FUS exon 6 (entire) $\boldsymbol{\nabla}$ insertion $\quad \boldsymbol{\nabla}$ CREB3L2 exon 5

1454-04 TGGAGGCAGAGGTGGCATGGGGTATGAGAAGCGTCGTGCCAACCCACGCCTGCACCCCTTCAGCCTGCCTCAGACCCACAGCCCCTCCAG $\begin{array}{lllllllllllllllllllllllllllllllll}G & G & R & G & G & M & G & Y & E & K & R & R & A & N & P & R & L & H & P & F & S & L & P & Q & T & H & S & P & S & P\end{array}$

FUS exon 6 (entire) $\quad \boldsymbol{\nabla}$ CREB3L2 exon 5

1455-04 AGGTCGTGGAGGTGGCCGTGGAGGCAGAGGTGGCATGGGCCTGAGTCCCAACCCACGCCTGCACCCCTTCAGCCTGCCTCAGACCCACAG $\begin{array}{llllllllllllllllllllllllllllllllll}G & R & G & G & G & R & G & G & R & G & G & M & G & L & S & P & N & P & R & L & H & P & F & S & L & P & Q & T & H & S\end{array}$

FUS exon 6 CREB3L2 intron 5

1818-04 GCAGCGGTGGCTATGGACAGCAGGACCGTGGAGGCCGCGCAGTGGCTTCTTCAGTGACTTTTCAAAATACTGCCTTTTGCTTAGAATGCA

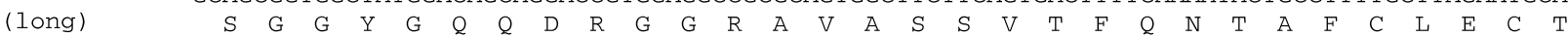
$\boldsymbol{V} \quad$ CREB3L2 exon 6

CTCCTCTCTCTTATAAACTGCAGGGATCAGGCCCTCTGGTCCTGACAGAGGAGGAGAAGAGGACCCTTCG

$\begin{array}{lllllllllllllllllllllll}P & L & S & Y & K & L & Q & G & S & G & P & L & V & L & T & E & E & E & K & R & T & L\end{array}$

FUS exon 5 V CREB3L2 intron 5

1818-04 TGGGAGCTACAGCCAGCAGCCTAGCTATGGTGGAGGCCGCGCAGTGGCTTCTTCAGTGACTTTTCAAAATACTGCCTTTTGCTTAGAATG

$\begin{array}{llllllllllllllllllllllllllllllllllll}\text { (short) } & G & S & Y & S & Q & Q & P & S & Y & G & G & G & R & A & V & A & S & S & V & T & F & Q & N & T & A & F & C & L & E & C\end{array}$

CREB3L2 exon 6

CACTCCTCTCTCTTATAAACTGCAGGGATCAGGCCCTCTGGTCCTGACAGAGGAGGAGAAGAGGACCCTTCG

$\begin{array}{llllllllllllllllllllllllllllll}\text { T } & P & L & S & Y & K & L & Q & G & S & G & P & \text { L } & \text { V } & \text { L } & \text { T } & \text { E } & \text { E } & \text { E } & \text { K } & \text { R } & \text { T } & \text { L }\end{array}$

FUS exon 6 (entire) $\quad \boldsymbol{\nabla}$ CREB3L2 exon 5

1819-04 GGTCGTGGAGGTGGCCGTGGAGGCAGAGGTGGCATGGGTGACTCAGAGGGCAGCCTGAGTCCCAACCCACGCCTGCACCCCTTCAGCCTG $\begin{array}{lllllllllllllllllllllllllllllll}G & R & G & G & G & R & G & G & R & G & G & M & G & D & S & E & G & S & L & S & P & N & P & R & L & H & P & F & S & L\end{array}$

FUS exon 6 (entire) $\boldsymbol{\nabla} \quad$ FUS intron $6 \quad \boldsymbol{\nabla}$ CREB3L2 exon 5

1820-04 TGGAGGCAGAGGTGGCATGGGCTTCCAGGAATTGGCTACTCTTCCCCACGGCAGTGACTCAGAGGGCAGCCTGAGTCCCAACCCACGCCT $\begin{array}{lllllllllllllllllllllllllllllll}G & G & R & G & G & M & G & F & Q & E & L & A & T & L & P & H & G & S & D & S & E & G & S & L & S & P & N & P & R\end{array}$

$\begin{array}{cccc}\text { FUS exon } 6 & \boldsymbol{\nabla} & \text { CREB3L2 exon } 5 \\ 1905-04 & \text { GGCGGCGGTGGTGGTACAACCGCAGCAGTGGTGGCTATGAACCACGCCTGCACCCCTTCAGCCTGCCTCAGACCCACAGCCCCTCCAGA }\end{array}$ $\begin{array}{llllllllllllllllllllllllllllllll}G & G & G & G & G & Y & N & R & S & S & G & G & Y & E & P & R & L & H & P & F & S & L & P & Q & T & H & S & P & S & R\end{array}$

FUS exon 6 (entire) $\quad$ VREB3L2 exon 5 CGTGGAGGTGGCCGTGGAGGCAGAGGTGGCATGGGCCTGCCTCAGACCCACAGC $\begin{array}{llllllllllllllllllll}R & G & G & G & R & G & G & R & G & G & M & G & L & P & Q & T & H & S\end{array}$ $\begin{array}{cccc}\text { FUS exon } 7 & \text { (entire) } & \boldsymbol{\nabla} & \text { CREB3L2 exon } \\ \text { GCGGAAGTGACCGTGGTGGCTTCAATAAATTTGGTGAGGGCAGCCTGAGTCCC }\end{array}$

$\begin{array}{lllllllllllllllllllllll}\text { G } & S & D & R & G & G & F & N & K & F & G & G & G & S & L & S & P\end{array}$

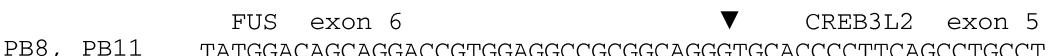
CREBTCAGCCTGCCT

$\begin{array}{llllllllllllllllllllllll}Y & G & Q & Q & D & R & G & G & R & G & R & V & H & P & F & S & L & P\end{array}$

PB13 FUS exon 7 省 CREB3L2 exon 5 CGGAAGTGACCGTGGTGGCTTCAATCCCAACCCACGCCTGCACCCCTTCAGCCTG $\begin{array}{llllllllllllllllllll}G & S & D & R & G & G & F & N & P & N & P & R & L & H & P & F & S & L\end{array}$

Figure 2 Partial nucleotide sequences spanning the breakpoints in the previously unreported FUS/CREB3L2 fusion transcripts. Arrowheads indicate site of junction. The predicted amino-acid sequences are written in capital letters. 


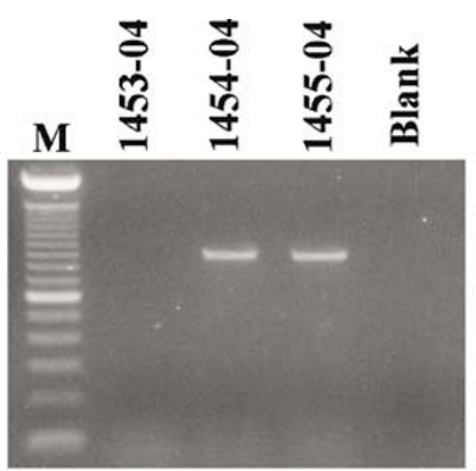

$1^{\text {st }}$ PCR with primers TLS165F and BBF2-1435R

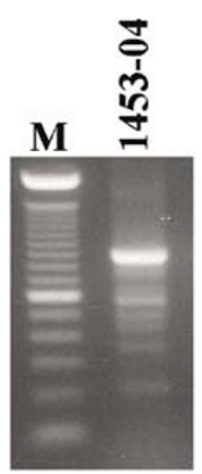

$2^{\text {nd }}$ PCR with primers TLS263F and BBF2-1396R

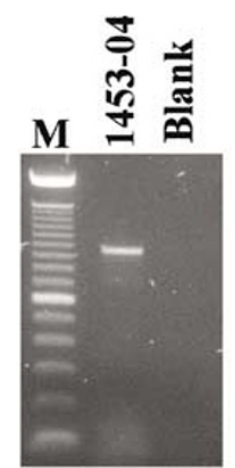

$1^{\text {st }}$ PCR with primers TLS165F and OASIS898R

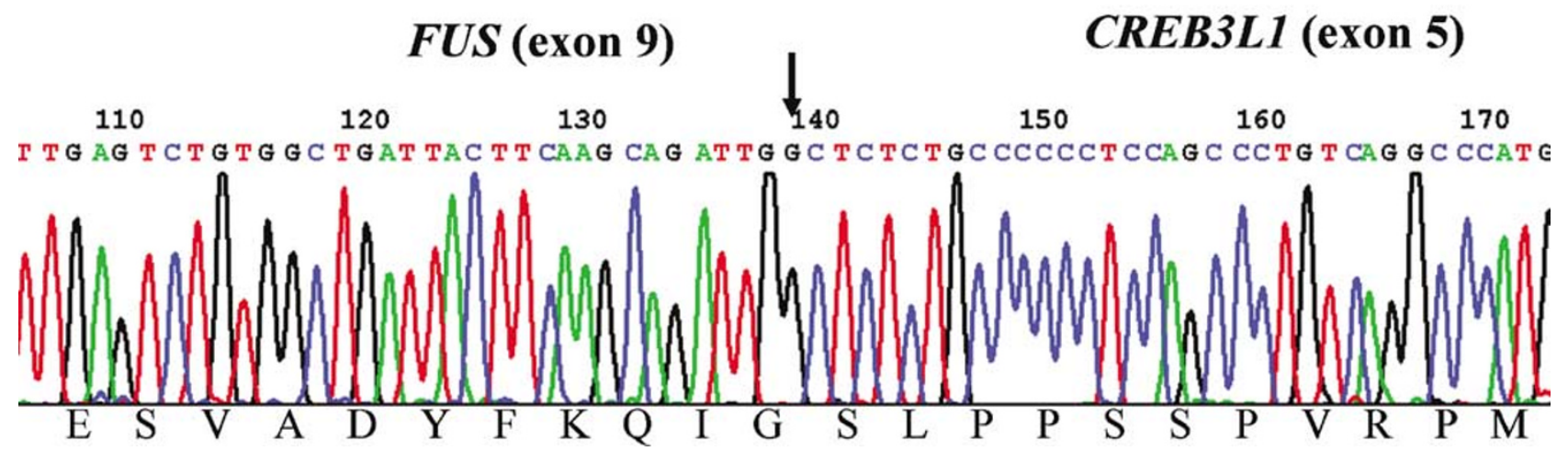

Figure 3 RT-PCR in case 1453/04 showing only a faint band with primer combination TLS165F and BBF2-1435R, one strong and several weaker bands with primers TLS263F and BBF2-1396R, and one strong, specific band with primer combination TLS165 and OASIS898R (upper part). Sequencing of the latter band showed a junction of FUS exon 9 with CREB3L1 exon 5. The predicted amino acid sequence is written in capital letters (lower part).

resulted in a faint amplified fragment, and a stronger band at second PCR with primers TLS263F and BBF2-1396R (Figure 3). Sequencing suggested that it represented a FUS/CREB3L1 fusion transcript instead of the expected FUS/CREB3L2 transcript. Thus, a renewed RT-PCR with primers specific for the CREB3L1 gene was performed, resulting in a strongly amplified fragment, which at direct sequencing could be shown to represent a FUS/CREB3L1 fusion transcript (Figure 3).

All 22 cases with the FUS/CREB3L2 fusion gene, as well as the case with the novel FUS/CREB3L1 chimera, were diagnosed as LGFMS at the histopathologic review. Of the six fusion-negative tumors, only one was considered a possible LGFMS; the remaining five cases were considered to represent other tumor types (data not shown).

\section{Discussion}

The main objective of the present study was to evaluate the incidence of the FUS/CREB3L2 fusion gene in LGFMS. A second aim was to search for alternative fusion genes in those cases that turned out to be negative for the FUS/CREB3L2 chimera. Several other sarcoma types in which characteristic chromosomal translocations and their corresponding fusion genes have been detected display more or less pronounced genetic heterogeneity, often with one type of fusion gene being much more common than the other(s). For instance, in Ewing's sarcoma the EWSR1/FLI1 fusion gene is by far the most frequent, accounting for approximately $85 \%$ of the cases, but some $10-15 \%$ of the tumors, morphologically indistinguishable from those which are EWSR1/FLI1-positive, are instead characterized by an EWSR1/ERG chimera. In addition, at least four other fusion genes, each representing less than $1 \%$ of the cases, have been detected. ${ }^{10}$ Similarly, four different fusion genes have been reported in synovial sarcoma and extraskeletal myxoid chondrosarcoma, three in alveolar rhabdomyosarcoma, and two in myxoid liposarcoma. ${ }^{11}$ From a clinical point of view, it is, of course, important to know whether a certain fusion gene is specific for a particular tumor type, or whether it might also be identified in other entities. Equally important, however, is to be aware of how often the tumor type might be fusionnegative or display an alternative genotype. For this 
purpose, a series of soft-tissue tumors that had been diagnosed by experienced pathologists as LGFMS or potential LGFMS were collected and subjected to molecular genetic analysis as well as histopathologic review. Overall, there was a very good correspondence between the histopathologic features and the molecular genetic findings. Of the 23 cases that remained classified as LGFMS after the histopathologic review, 22 (96\%) were found to be positive for the FUS/CREB3L2 fusion gene.

The remaining LGFMS in this series (case 1453/ 04) did not show rearrangement of the CREB3L2 gene, in spite of a typical morphologic appearance. Instead, we detected a novel fusion transcript corresponding to a chimeric FUS/CREB3L1 gene, consisting of the first nine exons of $F U S$ and exons 5-12 from CREB3L1. The CREB3L1 gene (cAMP responsive element-binding protein 3-like 1, also known as OASIS) is according to the Ensembl database located in chromosome band 11p11, consists of 12 exons, and encodes a protein of 528 amino acids. The protein is a transcription factor belonging to the OASIS basic-leucine zipper (B-ZIP) family of transcription factors together with CREB3L2, CREB-H, CREB3, and CREB4, all of which can dimerize with each other, but not with proteins belonging to other B-ZIP families. The strongest homology of CREB3L1 is seen with CREB3L2, the B-ZIP domain being $80 \%$ identical in these two proteins. ${ }^{7,12}$ Furthermore, both proteins are encoded by genes containing 12 exons, and in the FUS/ CREB3L1 chimera, just like in the FUS/CREB3L2 fusion protein, the B-ZIP-encoding domain is under the control of the FUS promoter.

The CREB3L1 gene has not previously been implicated in neoplasia-associated chromosome rearrangements, but bearing in mind the strong homology with CREB3L2, it is not entirely unexpected that it could serve as an alternative fusion partner to FUS in LGFMS. According to the Ensembl database, CREB3L1 is transcribed from telomere to centromere. As the FUS gene is transcribed in the same direction, the formation of the chimeric FUS/ CREB3L1 fusion gene would be achieved on the derivative chromosome 11 by a simple balanced translocation, in the same way as the FUS/CREB3L2 chimera is formed on the derivative chromosome 7 in cases with the $t(7 ; 16) .^{7}$ Thus, if the mapping of the CREB3L1 locus to band $11 \mathrm{p} 11$ is correct, the striking difference in frequency between the two fusion genes, the FUS/CREB3L2 chimera so far being 30 times more common, could not be explained by the need for additional genetic rearrangements in order to create a functional FUS/CREB3L1 fusion gene. However, it could be noted that other databases, for example, LocusLink, map the CREB3L1 locus to $11 \mathrm{q} 11$.

Combining the results of the present study with previously published data there is now information on FUS/CREB3L2 fusion transcripts in 31 cases of LGFMS. ${ }^{4,7}$ The most common type of FUS/CREB3L2 transcript among these tumors has been the ex6/ex5 fusion, seen in 15 cases, followed by the ex7/ex5 chimera, seen in 10 cases. Thus, the great majority of the cases have breakpoints within a rather restricted region of the two genes. However, as demonstrated in the present study, the fusion points in FUS and CREB3L2 may vary from exon 5 to exon 7 and from intron 4 to exon 6 , respectively. The case with the variant FUS/CREB3L1 fusion also had a break in exon 5 of the CREB gene, but as the breakpoint in FUS was in exon 9, a larger portion of FUS was included in the fusion gene.

Whether the variation in transcript type or size is of any clinical significance is presently unknown, but no apparent genotype-phenotype correlation could be detected in the present series; of the six patients who developed metastases, four had the ex6/ex5 type of transcript, one had an ex7/ex5, and one had an in6/ex5 transcript.

In summary, the present study demonstrates that RT-PCR for FUS/CREB3L2 constitutes an excellent tool in the differential diagnosis of LGFMS, as this fusion gene seems to account for more than $95 \%$ of the cases. However, our results also show that at least one alternative fusion gene, that is, FUS/ CREB3L1, may be associated with a small subset of LGFMS.

\section{Acknowledgements}

We thank Margareth Isaksson for excellent technical assistance. This work was supported by the Swedish Cancer Society and the Swedish Children's Cancer Foundation.

\section{References}

1 Evans HL. Low-grade fibromyxoid sarcoma. A report of two metastasizing neoplasms having a deceptively benign appearance. Am J Clin Pathol 1987;88:615-619.

2 Folpe AL, Lane KL, Paull G, et al. Low-grade fibromyxoid sarcoma and hyalinizing spindle cell tumor with giant rosettes: a clinicopathologic study of 73 cases supporting their identity and assessing the impact of high-grade areas. Am J Surg Pathol 2000; 24:1353-1360.

3 Folpe A, van den Berg E, Molenaar WM. Low grade fibromyxoid sarcoma. In: Fletcher CDM, Unni KK, Mertens F (eds). World Health Organization Classification of Tumours. Pathology and Genetics of Tumours of Soft Tissue and Bone. IARC Press: Lyon, 2002, pp 104-105.

4 Panagopoulos I, Storlazzi CT, Fletcher CDM, et al. The chimeric FUS/CREB3L2 gene is specific for low-grade fibromyxoid sarcoma. Genes Chromosomes Cancer 2004;40:218-228.

5 Bejarano PA, Padhya TA, Smith R, et al. Hyalinizing spindle cell tumor with giant rosettes-a soft tissue tumor with mesenchymal and neuroendocrine features. Arch Pathol Lab Med 2000;124:1179-1184. 
6 Reid R, de Silva MVC, Paterson L, et al. Low-grade fibromyxoid sarcoma and hyalinizing spindle cell tumor with giant rosettes share a common t(7;16)(q34;p11) translocation. Am J Surg Pathol 2003; 27:1229-1236.

7 Storlazzi CT, Mertens F, Nascimento A, et al. Fusion of the FUS and $B B F 2 H 7$ genes in low grade fibromyxoid sarcoma. Hum Mol Genet 2003;12:2349-2358.

8 Evans HL. Low-grade fibromyxoid sarcoma. A report of 12 cases. Am J Surg Pathol 1993;17:595-600.

9 Goodlad JR, Mentzel T, Fletcher CDM. Low-grade fibromyxoid sarcoma: a clinicopathological analysis of eleven new cases in support of a distinct entity. Histopathology 1995;26:229-237.
10 Ushigome S, Machinami R, Sorensen PH. Ewing sarcoma/primitive neuroectodermal tumour (PNET). In: Fletcher CDM, Unni KK, Mertens F (eds). World Health Organization Classification of Tumours. Pathology and Genetics of Tumours of Soft Tissue and Bone. IARC Press: Lyon, 2002, pp 298-300.

11 Mitelman F, Johansson B, Mertens F (eds). Mitelman Database of Chromosome Aberrations in Cancer. 2004; http://cgap.nci.nih.gov/Chromosomes/Mitelman.

12 Omori Y, Imai J, Suzuki Y, et al. OASIS is a transcriptional activator of CREB/ATF family with a transmembrane domain. Biochem Biophys Res Commun 2002;293:470-477. 